(2) Open Access Full Text Article

\title{
Anatomical and functional response after conversion to aflibercept using the treat- and-extend regimen protocol in bevacizumab treatment-resistant wet age-related macular degeneration
}

This article was published in the following Dove Press journal:

Clinical Ophthalmology

\author{
Claudia Taipale ${ }^{1,2}$ \\ Ilkka Laine ${ }^{1,3}$ \\ Raimo Tuuminen ${ }^{1,3}$ \\ 'Helsinki Retina Research \\ Group, Medicum, Faculty of \\ Medicine, University of Helsinki, \\ Helsinki, Finland; ' 2 Department of \\ Ophthalmology, Helsinki University \\ Hospital, Helsinki, Finland; \\ ${ }^{3}$ Department of Ophthalmology, \\ Kymenlaakso Central Hospital, \\ Kotka, Finland
}

Objective: To evaluate the functional and anatomical response after the switch from bevacizumab to aflibercept in treatment-resistant wet age-related macular degeneration (wAMD) using the treat-and-extend regimen protocol.

Design: A retrospective single-center study.

Participants: The registry consisted of 576 patients with wAMD. Of these, a total of 41 eyes of 37 patients met the study inclusion criteria with a minimum of three prior bevacizumab injections and at least 1-year follow-up after the switch to aflibercept injections for the treatment of wAMD.

Methods: Central retinal thickness (CRT) and best-corrected visual acuity (BCVA) were recorded before and after bevacizumab loading phase, before the switch to aflibercept, after aflibercept loading phase, and after the last injection or at the study end point at a minimum of 1 year from the switch.

Results: At the switch to aflibercept injections, the mean CRT was $361.1 \pm 117.7 \mu \mathrm{m}$ (mean $\pm \mathrm{SD}$ ) and BCVA was $0.29 \pm 0.19$ decimals. The switch to aflibercept resulted in mean CRT resolution by $59.9 \pm 80.2 \mu \mathrm{m}$ after the loading phase and by $61.3 \pm 102.9 \mu \mathrm{m}$ at the study end point. Anatomical response to aflibercept switch was found in 34 of 41 eyes (83\%) after the loading phase, and in 32 of 41 eyes $(78 \%)$ at the study end point. BCVA improvement was $0.08 \pm 0.13$ decimals in 26 of 41 eyes (63\%) after the loading phase, and $0.04 \pm 0.17$ decimals in 17 of 41 eyes ( $41 \%)$ at the study end point. The mean treatment interval of aflibercept was $8.0 \pm 2.2$ weeks at the study end point.

Conclusion: Regardless of impressive anatomical outcomes of aflibercept switch, functional response was modest for most of the study eyes at long term.

Keywords: aflibercept, anti-VEGF, bevacizumab, treat-and-extend regimen protocol, wet age-related macular degeneration

\section{Introduction}

Age-related macular degeneration (AMD) is a common cause for irreversible visual impairment and blindness among the elderly. ${ }^{1,2}$ The wet age-related macular degeneration (wAMD) usually leads to a rapid loss of vision and is characterized by intraretinal or subretinal fluid and hemorrhage. ${ }^{3}$ Vascular endothelial growth factor (VEGF) plays a major role in the pathogenesis of wAMD by promoting angiogenesis and vascular
Correspondence: Raimo Tuumin Kymenlaakso Central Hospital, Unit of Ophthalmology, Kotkantie 4I, Fl-482 10 Kotka, Finland Tel +35850 4II 3870 Fax +358924I 1227

Email raimo.tuuminen@helsinki.fi $\mathrm{BY}$
hC for commercial use of this work, please see paragraphs 4.2 and 5 of our Terms (https://wwww.dovepress.com/terms.php). 
permeability. Anti-VEGF agents given by intravitreal injections have become the standard of care in wAMD. ${ }^{4}$

The efficacy of anti-VEGF injections in the treatment of wAMD was first demonstrated with ranibizumab in two trials. Monthly treatment with ranibizumab was found to prevent vision loss and even improve visual acuity (VA) in some patients. ${ }^{5,6}$ Bevacizumab was found to have a similar efficacy and safety compared to ranibizumab, and is now widely used off-label as the first-line therapy in treating patients with wAMD. ${ }^{7,8}$ Aflibercept is a newer recombinant fusion protein. Compared with other anti-VEGF agents, aflibercept has a considerably greater binding affinity to VEGF and it also binds placental growth factor (PlGF). ${ }^{9}$ Treatment with aflibercept every 8 weeks has been shown to provide equal gain in VA compared to monthly treatment with ranibizumab. ${ }^{10}$ A recent meta-analysis demonstrated positive anatomical results with aflibercept in patients resistant to previous treatment with another anti-VEGF agent. ${ }^{11}$

The treatment protocols vary among different studies. A majority of earlier studies have used a fixed-interval treatment protocol with monthly injections, ${ }^{5,6}$ or a pro re nata regimen, where injections are given only when signs of active disease are observed. ${ }^{7,8}$ Both protocols require monthly visits, which burdens both the patient and the healthcare system. In the treat-and-extend regimen (TER) protocol, the objective is to gradually extend the treatment and follow-up interval while trying to avoid relapses of the disease. Many studies have shown equal visual gain with fewer injections using the TER protocol compared with monthly injections. ${ }^{12-14}$

In this study, we present results on the efficacy of aflibercept using the TER protocol in patients with wAMD resistant to previous treatment with bevacizumab. We evaluated the functional and anatomical response by measuring VA and central retinal thickness (CRT) before and after the switch from bevacizumab to aflibercept.

\section{Methods}

\section{Study design}

The study design was an institutional, retrospective, register-based, observational study. The study protocol was evaluated by the Institutional Review Board of Helsinki University Hospital. The study was approved by the Research Director and Chief Medical Officer of the Kymenlaakso Central Hospital and conducted according to the Declaration of Helsinki.

Patients were admitted for the management of wAMD to the Department of Ophthalmology, Kymenlaakso Central
Hospital, Kotka, Finland. All eyes with wAMD included in the retrospective analysis had a minimum of three prior bevacizumab (Avastin ${ }^{\circledR}$; Genentech, Inc., South San Francisco, CA, USA) injections $(1.25 \mathrm{mg} / 0.05 \mathrm{~mL})$, a minimum of three aflibercept $\left(\right.$ Eylea $^{\circledR}$; Bayer Leverkusen, Leverkusen, Germany) injections ( $2 \mathrm{mg} / 0.05 \mathrm{~mL})$, a minimum of 1-year follow-up after the switch to aflibercept and were treatmentnaive for aflibercept at the time of the switch. None of the eyes were treated with ranibizumab (Lucentis ${ }^{\circledR}$; Novartis, Basel, Switzerland) at any time. The diagnosis of wAMD was carried out by a physician specialized in its diagnosis and treatment, and all cases were treated using the TER protocol regardless of the anti-VEGF agent.

Bevacizumab was the first-line anti-VEGF agent in the treatment of WAMD. The choice of therapies between different anti-VEGF agents (bevacizumab or aflibercept) was at the discretion of the treating physician. Outcome measures were CRT and best-corrected visual acuity (BCVA) at baseline before the first bevacizumab intravitreal injection, after the bevacizumab loading phase, before the switch to aflibercept, after the aflibercept loading phase, and at termination of the treatment and/or follow-up at least after 1 year from the switch. The proportion of the eyes responding to the conversion to aflibercept was recorded. The final treatment interval with aflibercept was also determined.

This study was conducted by monitoring the clinical practice. The study was approved by the Research Director and Chief Medical Officer of the Kymenlaakso Central Hospital. Confidentiality of the patient records was maintained while entering the clinical data to a computer-based standardized database for analysis.

\section{Participant criteria}

The source of patients comprised the Kymenlaakso Health Care District Population and as such was representative of the entire source population $(\mathrm{n}=174,000)$. The registry consisted of 576 patients with wAMD (ICD code: H35.31) who were given intravitreal injections (ICD code: CKD05) between January 1, 2011 and September 30, 2016. Follow-up of the patients was terminated on September 30, 2017. Of these, a total of 41 eyes of 37 patients met the study inclusion criteria which are as follow: 1) a minimum of three prior bevacizumab injections, 2) a minimum of three aflibercept injections after the switch, 3) treatment naïve for aflibercept at the time of the switch, 4) no ranibizumab treatment at any time, and 5) a minimum of 1 year follow-up after the switch to aflibercept. 
The treated eye was considered a nonresponder to the drug, when observing unchanged or increased CRT within three consecutive intravitreal injections with the shortest 4-week treatment interval.

The baseline variables are presented in Table 1 .

\section{Clinical evaluation}

The diagnosis of wAMD was carried out by a physician specialized in its diagnosis and treatment. Clinical examination included bilateral VA testing, biomicroscopy, tonometry, and examination of the fundus. Fluorescein angiography was performed when necessary. Initiation of anti-VEGF treatment was performed on the day of the diagnosis in most of the cases.

The CRT was recorded by spectral-domain optical coherence tomography (OCT; Heidelberg Engineering $\mathrm{GmbH}$, Heidelberg, Germany) by an experienced ophthalmic nurse. Follow-up 30-frame scans were performed with AutoRescan ${ }^{\mathrm{TM}}$ software, and the OCT analyses were compared to the previous ones (Heidelberg Eye Explorer Version 1.9.10.0 and HRA/SPECTRALIS ${ }^{\circledR}$ Viewing Module Version 6.0.9.0; Heidelberg Engineering $\mathrm{GmbH}$ ).

The eligibility of the patient for treatment with aflibercept was estimated with the aid of guiding principles. These included no subretinal fibrosis, no geographic atrophy in the fovea, and a BCVA of 0.1 decimals or more in the treated eye. However, the treatment decision was always made case-by-case taking all the patient characteristics into account.

Discontinuation of the anti-VEGF treatment was discussed with the patient, in case BCVA was $<0.0625$ decimals in the treated eye (when necessary, confirmed with below

Table I Baseline variables of 4 I eyes with wAMD of 37 patients not responding to bevacizumab

\begin{tabular}{l|l}
\hline & Nonresponders \\
\hline Male:female (n/\%) & $12: 25(32: 68)$ \\
\hline Age (years) & $81.0 \pm 5.4(70-93)$ \\
Laterality (uni:bilateral) & $22: 15^{\mathrm{a}}(59: 4 \mathrm{I})$ \\
Phakic:pseudophakic $(\mathrm{n} / \%)$ & $14: 27(34: 66)$ \\
BCVA (decimal units) & $0.29 \pm 0.14(0.05-0.60)$ \\
CRT mean $(\mu \mathrm{m})$ & $459.7 \pm 157.6(220-892)$ \\
CRT max $(\mu \mathrm{m})$ & $557.9 \pm 174.5(299-1,098)$ \\
\hline
\end{tabular}

Notes: Baseline variables regarding patient and ophthalmic parameters. Data are given as mean $( \pm S D)$ and range or absolute number and proportion. ${ }^{\mathrm{a}} \mathrm{Of}$ I 5 bilateral wAMD patients, 4 were bevacizumab nonresponders for both eyes; in the rest I I bilateral wAMD, the contralateral eye was treated with bevacizumab.

Abbreviations: BCVA, best-corrected visual acuity; CRT, central retinal thickness; wAMD, wet age-related macular degeneration.
20 Early Treatment Diabetic Retinopathy Study (ETDRS) letters), or if the treatment was not expected to enhance the patient's quality of life or ability to function.

In VA testing, when the largest optotype could not be recognized correctly, the classification was very low VA on a semiquantitative scale such as counting fingers $(\mathrm{CF})$ and hand motion (HM). The very low VA measurements have been converted as follows: CF to 1.9 and HM to 2.3 logarithm of minimum angle of resolution ( $\operatorname{logMAR}$ ) units. ${ }^{15}$ For statistical purposes, the $\log \mathrm{MAR}$ units for $\mathrm{CF}$ and $\mathrm{HM}$ were converted to the equivalent values of 0.013 and 0.005 decimals, respectively.

\section{TER protocol (anti-VEGF treatment protocol)}

Anti-VEGF treatment was initiated with three loading doses of the primary drug bevacizumab given approximately 4 weeks apart. After adequate treatment response was achieved with either bevacizumab or the secondary drug aflibercept, the treatment interval was lengthened gradually by 2 weeks up to 12 weeks. When intraretinal or subretinal fluid remained unchanged compared to the previous OCT scan, the treatment interval was maintained.

The treatment interval was shortened 2 weeks at a time if the examination showed any signs of recurrence, eg, when observing 1) any increase of intraretinal or subretinal fluid compared with the previous OCT scan, or 2) any increase in the size of pigment epithelial detachment compared with the previous OCT scan, or 3) any sign of exudation or new macular hemorrhage in slit-lamp biomicroscopic examination, or 4) worsening of subjective vision and BCVA in cases ambiguous by OCT. In case of a large recurrence of intra- or subretinal fluid associated with visual loss over 0.2 decimals, subfoveal or large extrafoveal hemorrhage, the treatment was reverted to monthly injections and then extended according to the treatment protocol. If a second recurrence was observed, the patient-specific maximum final interval was set at 2 weeks less than the period when the previous recurrence was observed.

The treatment intervals were recorded on every visit.

\section{Statistical analysis}

Data are given as mean \pm standard error of the mean (SEM) and range ( $\min -\max$ ) except for the absolute number and proportion for nominal scale. IBM SPSS Statistics 23 (SPSS Inc., Somers, NY, USA) was used for statistical analyses. A linear regression model was used to estimate the relationships between variables. $P \leq 0.05$ was considered statistically significant. 


\section{Results}

\section{Baseline patient and ophthalmic characteristics}

The gender distribution was 12 males (32\%) and 25 females $(68 \%)$. The mean age at the time of initiation of primary intravitreal treatment was 81 years (range 70-93 years). Of the 37 study patients, 22 (59\%) had unilateral and 15 (41\%) had bilateral disease. Of those 15 study patients with bilateral disease, 4 (27\%) were bevacizumab nonresponders for both eyes (Table 1 ).

Of the 41 study eyes, 14 (34\%) were phakic and 27 (66\%) were pseudophakic prior to initiation of the primary intravitreal treatment. The baseline mean BCVA was 0.29 decimals (range 0.05-0.60 decimals), CRT mean was $460 \mu \mathrm{m}$ (range 220-892 $\mu \mathrm{m}$ ), and CRT max was $558 \mu \mathrm{m}$ (range 299-1,098 $\mu \mathrm{m}$; Table 1).

\section{CRT change after the conversion to} aflibercept in bevacizumab nonresponder eyes in short and long terms

The mean CRT at baseline was $459.7 \pm 157.6 \mu \mathrm{m}$ (range 220-892 $\mu \mathrm{m})$. The corresponding values after bevacizumab loading phase with three injections and at the switch to

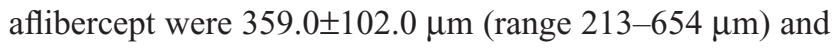
361.1 $\pm 117.7 \mu \mathrm{m}$ (range 206-654 $\mu \mathrm{m}$, respectively; Table 2).

The mean CRT after three aflibercept injections was 301.3 $\pm 88.7 \mu \mathrm{m}(163-544 \mu \mathrm{m})$. At the study termination, a minimum of 1 year after the conversion to aflibercept, the

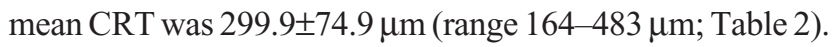

CRT change and final aflibercept treatment interval according to macular anatomical characteristics at the time of the switch to aflibercept are presented in Table 3.

\section{BCVA gain after the conversion to aflibercept in bevacizumab nonresponder eyes in short and long terms}

BCVA was 0.29 \pm 0.14 decimals (range 0.05-0.60 decimals) at baseline. The corresponding values after bevacizumab loading phase with three injections and at the switch to aflibercept were $0.30 \pm 0.19$ (range $0.05-0.80$ ) and $0.29 \pm 0.19$ (range 0.05-0.80) decimals, respectively (Table 4).

BCVA after three aflibercept injections was $0.36 \pm 0.24$ decimals (range $\mathrm{CF}-1.00$ decimals). At the study termination, at least 1 year after the conversion to aflibercept, BCVA was $0.33 \pm 0.23$ decimals (range HM-0.80 decimals; Table 4).

\section{The conversion to aflibercept results in visual acuity improvement for most eyes in short term but not in long term}

Next, we analyzed the proportion of eyes responding to aflibercept switch regarding VA and macular anatomy.

Compared to the clinical status before the switch to aflibercept, anatomical response was found in 34 of the 41 eyes (83\%) after three aflibercept injections. At the study termination, anatomical response to aflibercept was maintained in 32 of the 41 eyes (78\%).

After three aflibercept injections, BCVA improved in $26(63 \%)$, remained unchanged in $9(22 \%)$, and declined in 6 of the 41 eyes (15\%) compared with the BCVA before the conversion to aflibercept (Table 5).

At the study termination, an improved BCVA was maintained in 17 of 41 eyes (41\%). Eleven of the 41 eyes (27\%) remained unchanged and 13 eyes $(32 \%)$ had a declined BCVA compared with the BCVA before the switch to aflibercept (Table 5).

Irrespective of the fact that a dry macula was maintained with aflibercept injections in most of the patients, the distribution between eyes with improved, unchanged, or decreased BCVA was fairly even at the study end point. The change in CRT from aflibercept switch to that after the aflibercept loading phase $\left(R^{2}=0.028 ; P=0.299\right)$, and from aflibercept switch to study end point $\left(R^{2}=0.021 ; P=0.367\right)$ did not correlate with the change in BCVA at given time points.

Finally, we analyzed the treatment intervals at the study end point. Six of the 41 study eyes $(15 \%)$ were still in the extend phase of the TER protocol at the time point of the

Table 2 CRT of 4 I eyes with wet age-related macular degeneration not responding to bevacizumab

\begin{tabular}{l|l|l}
\hline & Mean & Max \\
\hline CRT at baseline & $459.7 \pm 157.6(220-892)$ & $557.9 \pm 174.5(299-1,098)$ \\
CRT after three bevacizumab injections & $359.0 \pm 102.0(213-654)$ & $435.9 \pm 117.0(265-725)$ \\
CRT at switch to aflibercept & $361.1 \pm 117.7(206-654)$ & $459.1 \pm 126.6(265-725)$ \\
CRT after three aflibercept injections & $301.3 \pm 88.7(163-544)$ & $387.1 \pm 100.2(256-638)$ \\
CRT final & $299.9 \pm 74.9(164-483)$ & $393.8 \pm 96.9(260-643)$ \\
\hline
\end{tabular}

Note: Data are given as mean $( \pm S D)$ and range.

Abbreviation: CRT, central retinal thickness. 
Table 3 Anatomical and treatment response according to macular status at switch to aflibercept

\begin{tabular}{l|l|l|l}
\hline $\begin{array}{l}\text { Macular status at switch } \\
\text { to aflibercept }\end{array}$ & $\begin{array}{l}\text { CRT change after three } \\
\text { aflibercept injections }(\mu \mathrm{m})\end{array}$ & CRT change final $(\mu \mathrm{m})$ & $\begin{array}{l}\text { Treatment interval } \\
\text { final }(\text { weeks) }\end{array}$ \\
\hline IRF & $5.0 \pm 37.9$ & $-17.7 \pm 25.3$ & $10.0 \pm 2.2$ \\
No $(\mathrm{N}=6)(I 5 \%)$ & $-71.0 \pm 80.6$ & $-68.7 \pm 109.4$ & $7.6 \pm 2.0$ \\
Yes $(\mathrm{N}=35)(85 \%)$ & $-30.2 \pm 50.2$ & $-32.9 \pm 81.7$ & $8.5 \pm 2.1$ \\
PED & $-80.9 \pm 91.3$ & $-81.3 \pm 112.9$ & $7.6 \pm 2.3$ \\
No $(\mathrm{N}=17)(41 \%)$ & & & $7.9 \pm 2.3$ \\
Yes $(\mathrm{N}=24)(59 \%)$ & $-46.4 \pm 68.2$ & $-46.2 \pm 99.7$ & $8.0 \pm 2.2$ \\
Subretinal fluid & $-71.4 \pm 89.2$ & $-74.2 \pm 106.0$ & \\
No $(\mathrm{N}=19)(46 \%)$ & & $-54.8 \pm 93.8$ & $8.0 \pm 2.3$ \\
Yes $(\mathrm{N}=22)(54 \%)$ & $-53.9 \pm 69.5$ & $-187.5 \pm 235.5$ & $8.0 \pm 0.0$ \\
\hline Subretinal fibrosis & $-176.0 \pm 213.5$ & \\
No $(\mathrm{N}=39)(95 \%)$ & & & \\
Yes $(\mathrm{N}=2)(5 \%)$ &
\end{tabular}

Note: Data are given as absolute numbers and proportions or mean $( \pm S D)$.

Abbreviations: CRT, central retinal thickness; IRF, intraretinal fluid; PED, pigment epithelial detachment.

analysis. The mean treatment interval with aflibercept was 8.0 \pm 2.2 weeks (range 4-12 weeks; Table 6).

\section{Discussion}

Since the introduction of aflibercept, it has become an alternative treatment option for eyes with wAMD resistant to treatment with other anti-VEGF agents. In eyes with treatment-resistant wAMD, the conversion to aflibercept may result in a positive functional and anatomical response. A recent meta-analysis demonstrated that the switch to aflibercept results in a significant improvement in CRT, but the effect on VA is far more modest. ${ }^{11}$

In this study, we found that treatment with aflibercept in accordance with the TER protocol improves the anatomy and decreases CRT in most of the eyes with persistent macular fluid despite previous treatment with bevacizumab. A positive anatomical response was found in $83 \%$ of the eyes after three aflibercept injections and in $78 \%$ of the eyes at the end of the study. The positive anatomical results are consistent with other studies evaluating the effect of aflibercept on eyes resistant to previous treatment with other anti-VEGF

Table 4 Visual acuity of $4 \mathrm{I}$ eyes with wet age-related macular degeneration not responding to bevacizumab

\begin{tabular}{l|l}
\hline & BCVA \\
\hline At baseline & $0.29 \pm 0.14(0.05-0.60)$ \\
\hline After three bevacizumab injections & $0.30 \pm 0.19(0.05-0.80)$ \\
At switch to aflibercept & $0.29 \pm 0.19(0.05-0.80)$ \\
After three aflibercept injections & $0.36 \pm 0.24(\mathrm{CF}-\mathrm{I} .00)$ \\
At study end point & $0.33 \pm 0.23(\mathrm{HM}-0.80)$ \\
\hline
\end{tabular}

Note: Data are given as mean $( \pm S D)$ and range.

Abbreviations: BCVA, best-corrected visual acuity; CF, counting fingers; HM, hand motion. agents. ${ }^{16-18}$ Compared with other anti-VEGF agents, aflibercept additionally binds PIGF and has a greater binding affinity to VEGF. ${ }^{9}$ Moreover, repeated injections with bevacizumab or ranibizumab can lead to immunoreactivity against mousederived humanized monoclonal antibodies and/or tachyphylaxis with loss of therapeutic effect, ${ }^{19-21}$ and switching to another anti-VEGF agent can overcome this issue. ${ }^{22}$

Irrespective of the fact that a dry macula was maintained with aflibercept injections throughout the study in most of the patients, the positive anatomical results did not correlate with restoration of visual function. The proportion of eyes with an improved, unchanged, or decreased BCVA was distributed fairly evenly at the end of the study. Compared with our data, some previous studies have reported a more significant functional response, ${ }^{16,17}$ and on the contrary, one study demonstrated a decrease in mean BCVA after a 2-year follow-up. ${ }^{18}$ Aflibercept is considerably more expensive than bevacizumab, which makes a question regarding the cost-effectiveness of the conversion to aflibercept. Of note, aflibercept switch may improve contrast sensitivity and thus vision-related quality of life, ${ }^{23}$ even without changes in BCVA. ${ }^{24}$

Table 5 Visual acuity outcome after switch to aflibercept of 4I wAMD eyes not responding to bevacizumab

\begin{tabular}{l|l|l}
\hline & $\begin{array}{l}\text { After three } \\
\text { aflibercept injections }\end{array}$ & $\begin{array}{l}\text { At study } \\
\text { end point }\end{array}$ \\
\hline BCVA gain $(\mathrm{N}=$ =eyes) & $26(63 \%)$ & $17(41 \%)$ \\
BCVA unchanged $(\mathrm{N}=$ =eyes) & $9(22 \%)$ & $11(27 \%)$ \\
BCVA decline $(\mathrm{N}=$ =eyes) & $6(15 \%)$ & $13(32 \%)$ \\
\hline
\end{tabular}

Notes: Data are given as the absolute number and proportion. The follow-up was at least I year from the switch to aflibercept.

Abbreviations: BCVA, best-corrected visual acuity; wAMD, wet age-related macular degeneration. 
Table 6 Aflibercept treatment interval of 4 I eyes with wet agerelated macular degeneration not responding to bevacizumab

\begin{tabular}{|l|l|}
\hline Treatment interval at end point (weeks) & $8.0 \pm 2.2^{\mathrm{a}}(4-12)$ \\
\hline
\end{tabular}

Notes: Data are given as mean $( \pm S D)$ and range. a ${ }^{2}$ ix eyes were on extend phase in the TER protocol at the time of analysis. The follow-up was at least I year from the switch to aflibercept.

Abbreviation: TER, treat-and-extend regimen.

It can be reasoned that the lack of significant functional response in long term might be related to progression of retinal atrophic changes. VEGF has an essential role in maintaining the cone photoreceptors and choroid vasculature, and it has been suggested that persistent VEGF antagonism in the eye might have detrimental side-effects. ${ }^{25}$ Anti-VEGF treatment has been associated with accelerated progression and development of geographic atrophy. ${ }^{26-28}$ One could argue that the functional unresponsiveness might be a result of permanent damage of the macula before the conversion to aflibercept. In that case, a minimal delay in the conversion of the treatment-resistant eyes to aflibercept could lead to better functional results.

This study has several limitations. First, the patients' baseline characteristics are heterogeneous regarding the severity of the disease and the duration of the previous treatment with bevacizumab. The decision of the treatment modality was nonrandomized and at the discretion of the treating clinician. This reflects the real-life setting of the study. The lack of a control group also makes the interpretation of the results more difficult. The strength of the study was its long follow-up to evaluate the effects of the switch to aflibercept also in the long run. The growing real-world evidence of the aflibercept switch on the anatomical, functional, and quality of life outcomes helps physicians to weigh the pros and cons of the switch for the individual.

In conclusion, this study added real-world evidence that most of the eyes resistant to treatment with bevacizumab expressed a positive anatomical response. In addition, treatment interval was doubled following the switch to aflibercept using the TER protocol. However, with poor correlation between the anatomical results and the BCVA improvement, further studies concentrating on other visual function parameters and quality of life outcomes are warranted.

\section{Acknowledgments}

The study was supported by grants from the Helsinki University Hospital Specific Catchment Area Clinical Research Grants, the Finnish Eye Foundation, Finnish Ophthalmological Society, the Nissi Foundation, the Paulo Foundation, and the Waldemar von Frenckell Foundation, Helsinki, Finland.

\section{Disclosure}

The authors report no conflicts of interest in this work.

\section{References}

1. Colijn JM, Buitendijk GHS, Prokofyeva E, et al. Prevalence of agerelated macular degeneration in Europe: the past and the future. Ophthalmology. 2017;124(12):1753-1763.

2. Congdon NG, Friedman DS, Lietman T. Important causes of visual impairment in the world today. JAMA. 2003;290(15):2057-2060.

3. Lim LS, Mitchell P, Seddon JM, Holz FG, Wong TY. Age-related macular degeneration. Lancet. 2012;379(9827):1728-1738.

4. Solomon SD, Lindsley K, Vedula SS, Krzystolik MG, Hawkins BS. Antivascular endothelial growth factor for neovascular age-related macular degeneration. Cochrane Database Syst Rev. 2014;8:Cd005139.

5. Rosenfeld PJ, Brown DM, Heier JS, et al. Ranibizumab for neovascular age-related macular degeneration. $N$ Engl J Med. 2006;355(14): 1419-1431.

6. Brown DM, Michels M, Kaiser PK, et al. Ranibizumab versus verteporfin photodynamic therapy for neovascular age-related macular degeneration: two-year results of the ANCHOR study. Ophthalmology. 2009;116(1):57.e5-65.e5.

7. Comparison of Age-related Macular Degeneration Treatments Trials (CATT) Research Group, Martin DF, Maguire MG, et al. Ranibizumab and bevacizumab for treatment of neovascular age-related macular degeneration: two-year results. Ophthalmology. 2012;119(7): $1388-1398$.

8. Chakravarthy U, Harding SP, Rogers CA, et al. Alternative treatments to inhibit VEGF in age-related choroidal neovascularisation: 2-year findings of the IVAN randomised controlled trial. Lancet. 2013;382(9900):1258-1267.

9. Holash J, Davis S, Papadopoulos N, et al. VEGF-Trap: a VEGF blocker with potent antitumor effects. Proc Natl Acad Sci U S A. 2002;99(17): 11393-11398.

10. Heier JS, Brown DM, Chong V, et al. Intravitreal aflibercept (VEGF trap-eye) in wet age-related macular degeneration. Ophthalmology. 2012;119(12):2537-2548

11. Seguin-Greenstein S, Lightman S, Tomkins-Netzer O. A meta-analysis of studies evaluating visual and anatomical outcomes in patients with treatment resistant neovascular age-related macular degeneration following switching to treatment with aflibercept. J Ophthalmol. 2016; 2016:1-8.

12. Wykoff CC, Croft DE, Brown DM, et al. Prospective trial of treatand-extend versus monthly dosing for neovascular age-related macular degeneration: TREX-AMD 1-year results. Ophthalmology. 2015; 122(12):2514-2522.

13. Silva R, Berta A, Larsen M, et al. Treat-and-extend versus monthly regimen in neovascular age-related macular degeneration: results with ranibizumab from the TREND Study. Ophthalmology. 2018;125(1): $57-65$.

14. Berg K, Roald AB, Navaratnam J, Bragadóttir R. An 8-year follow-up of anti-vascular endothelial growth factor treatment with a treat-andextend modality for neovascular age-related macular degeneration. Acta Ophthalmol. 2017;95(8):796-802.

15. Schulze-Bonsel K, Feltgen N, Burau H, Hansen L, Bach M. Visual acuities "hand motion" and "counting fingers" can be quantified with the freiburg visual acuity test. Invest Ophthalmol Vis Sci. 2006;47(3): $1236-1240$.

16. Chang AA, Li H, Broadhead GK, et al. Intravitreal aflibercept for treatment-resistant neovascular age-related macular degeneration. Ophthalmology. 2014;121(1):188-192.

17. Singh RP, Srivastava S, Ehlers JP, Bedi R, Schachat AP, Kaiser PK. A single-arm, investigator-initiated study of the efficacy, safety and tolerability of intravitreal aflibercept injection in subjects with exudative age-related macular degeneration, previously treated with ranibizumab or bevacizumab: 6-month interim analysis. Br J Ophthalmol. 2014; 98(Suppl 1):i22-i27. 
18. Jørstad ØK, Faber RT, Moe MC. Two-year functional and anatomical results after converting treatment resistant eyes with exudative agerelated macular degeneration to aflibercept in accordance with a treat and extend protocol. Acta Ophthalmol. 2017;95(5):460-463.

19. Forooghian F, Cukras C, Meyerle CB, Chew EY, Wong WT. Tachyphylaxis after intravitreal bevacizumab for exudative age-related macular degeneration. Retina. 2009;29(6):723-731.

20. Keane PA, Liakopoulos S, Ongchin SC, et al. Quantitative subanalysis of optical coherence tomography after treatment with ranibizumab for neovascular age-related macular degeneration. Invest Ophthalmol Vis Sci. 2008;49(7):3115-3120.

21. Schaal S, Kaplan HJ, Tezel TH. Is there tachyphylaxis to intravitreal anti-vascular endothelial growth factor pharmacotherapy in age-related macular degeneration? Ophthalmology. 2008;115(12):2199-2205.

22. Gasperini JL, Fawzi AA, Khondkaryan A, et al. Bevacizumab and ranibizumab tachyphylaxis in the treatment of choroidal neovascularisation. Br J Ophthalmol. 2012;96(1):14-20.

23. Zhu M, Wijeyakumar W, Syed AR, et al. Vision-related quality of life: 12-month aflibercept treatment in patients with treatment-resistant neovascular age-related macular degeneration. Graefes Arch Clin Exp Ophthalmol. 2017;255(3):475-484.
24. Nixon DR, Flinn NA. Evaluation of contrast sensitivity and other visual function outcomes in neovascular age-related macular degeneration patients after treatment switch to aflibercept from ranibizumab. Clin Ophthalmol. 2017;11:715-721.

25. Kurihara T, Westenskow PD, Bravo S, Aguilar E, Friedlander M. Targeted deletion of Vegfa in adult mice induces vision loss. J Clin Invest. 2012;122(11):4213-4217.

26. Grunwald JE, Pistilli M, Daniel E, et al. Incidence and growth of geographic atrophy during 5 years of comparison of age-related macular degeneration treatments trials. Ophthalmology. 2017;124(1):97-104.

27. Grunwald JE, Pistilli M, Ying GS, et al. Growth of geographic atrophy in the comparison of age-related macular degeneration treatments trials. Ophthalmology. 2015;122(4):809-816.

28. Young M, Chui L, Fallah N, et al. Exacerbation of choroidal and retinal pigment epithelial atrophy after anti-vascular endothelial growth factor treatment in neovascular age-related macular degeneration. Retina. 2014;34(7):1308-1315.
Clinical Ophthalmology

\section{Publish your work in this journal}

Clinical Ophthalmology is an international, peer-reviewed journa covering all subspecialties within ophthalmology. Key topics include: Optometry; Visual science; Pharmacology and drug therapy in eye diseases; Basic Sciences; Primary and Secondary eye care; Patient Safety and Quality of Care Improvements. This journal is indexed on

Submit your manuscript here: http://www.dovepress.com/clinical-ophthalmology-journal

\section{Dovepress}

PubMed Central and CAS, and is the official journal of The Society of Clinical Ophthalmology (SCO). The manuscript management system is completely online and includes a very quick and fair peer-review system, which is all easy to use. Visit http://www.dovepress.com/ testimonials.php to read real quotes from published authors. 